Revue québécoise de psychologie

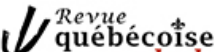

de psychologie

\title{
LE SYNDROME DE GILLES DE LA TOURETTE EN TANT QUE TROUBLE NEURODÉVELOPPEMENTAL TOURETTE SYNDROME AS A NEURODEVELOPMENTAL DISORDER
}

\author{
Julie B. Leclerc, Isabelle Gaudet, Bruno Gauthier et Juliette St-Denis-Turcotte
}

Volume 37, numéro 2, 2016

LES TROUBLES NEURODÉVELOPPEMENTAUX

NEURODEVELOPMENTAL DISORDERS

URI : https://id.erudit.org/iderudit/1040039ar

DOI : https://doi.org/10.7202/1040039ar

Aller au sommaire du numéro

Éditeur(s)

Revue québécoise de psychologie

ISSN

2560-6530 (numérique)

Découvrir la revue

Citer cet article

Leclerc, J. B., Gaudet, I., Gauthier, B. \& St-Denis-Turcotte, J. (2016). LE

SYNDROME DE GILLES DE LA TOURETTE EN TANT QUE TROUBLE

NEURODÉVELOPPEMENTAL. Revue québécoise de psychologie, 37(2), 97-120.

https://doi.org/10.7202/1040039ar
Résumé de l'article

L'objet de cet article est de présenter les avancées scientifiques concernant le syndrome de Gilles de la Tourette (SGT), dans l'optique où il fait partie de la nouvelle section des troubles neurodéveloppementaux du DSM-5. La définition et la symptomatologie des troubles tic, et particulièrement du SGT, sont présentées. Les troubles associés, ainsi que l'évaluation diagnostique et différentielle sont abordés sous l'angle clinique et neuropsychologique. Enfin, les traitements recommandés, et s'appuyant sur des données probantes, sont présentés de manière à outiller les professionnels et les psychologues, tout en les informant des principaux modèles conceptuels permettant une meilleure compréhension clinique des tics. 


\title{
LE SYNDROME DE GILLES DE LA TOURETTE EN TANT QUE TROUBLE NEURODÉVELOPPEMENTAL
}

\author{
TOURETTE SYNDROME AS A NEURODEVELOPMENTAL DISORDER \\ Julie B. Leclerc ${ }^{1}$ \\ Isabelle Gaudet \\ Université du Québec à Montréal \\ Université de Montréal \\ Bruno Gauthier \\ Université de Montréal \\ Juliette St-Denis-Turcotte \\ Université du Québec à Montréal
}

\section{INTRODUCTION}

\section{Du DSM-IV-TR au DSM-5}

La $5^{\mathrm{e}}$ version du Manuel diagnostique et statistique des troubles mentaux parue en 2013 (DSM-5; American Psychiatric Association, 2013) révèle des changements importants par rapport au DSM-IV-TR (APA, 2000), ce qui nécessite une adaptation clinique et conceptuelle de la part des psychologues et autres professionnels qui utilisent l'ouvrage de référence. Ces modifications visent dans un premier temps à adopter une approche dimensionnelle, considérant certains troubles en termes de continuum ou de spectres cliniques, et ainsi, des niveaux d'atteintes sont ajoutés impliquant une gradation du soutien nécessaire. Par ailleurs, les changements ont été réalisés dans l'objectif d'une harmonisation internationale (p. ex., Classification Internationale des Maladies) et d'une structure plus fidèle aux données probantes et à l'état des connaissances scientifiques. Ainsi, la classification multiaxiale a été modifiée en combinant les axes I, II et III et en supprimant les axes IV et V, de nombreux troubles se sont ainsi vu attribuer de nouvelles catégories. De nombreux changements apportés concernent la catégorie des troubles diagnostiqués pendant la première enfance, la deuxième enfance ou l'adolescence puisque cette catégorie a été supprimée dans le DSM-5. Certains troubles dont la déficience intellectuelle, les troubles spécifiques des apprentissages, les troubles de la communication, les troubles du spectre de l'autisme, le trouble déficitaire de l'attention avec hyperactivité et les troubles moteurs (comprenant les troubles tic) figurent maintenant dans la catégorie des troubles neurodéveloppementaux. Les troubles faisant partie de cette catégorie sont caractérisés par un déclenchement au cours du développement de l'individu, la plupart du temps dans l'enfance. Ils engendrent des retards développementaux qui nuisent au fonctionnement personnel, social, académique et occupationnel de

1. Adresse de correspondance : Université du Québec à Montréal, Centre de recherche de l'Institut universitaire en santé mentale de Montréal, 7331, rue Hochelaga, Montréal (QC), H1N 3V2. Téléphone : 514-251-4015, poste 2340. Courriel : leclerc.julie@uqam.ca 
l'individu et ils sont régulièrement caractérisés par de nombreuses comorbidités (APA, 2013).

\section{Troubles tic}

La section des troubles neurodéveloppementaux du DSM- 5 comporte une rubrique "troubles moteurs" qui inclut les troubles tic. L'ensemble des tics regroupe le trouble tic spécifié/non spécifié, le trouble tic provisoire, le trouble tic moteur ou sonore persistant (chronique), ainsi que le syndrome de Gilles de la Tourette (APA, 2013). Ces troubles figuraient dans la section des troubles diagnostiqués dans l'enfance et l'adolescence du DSM-IV-TR (APA, 2000) et les symptômes devaient être présents avant l'âge de 18 ans. Cet aspect clinique demeure pour chaque trouble tic, malgré le changement de classification. D'ailleurs, les critères diagnostiques des troubles tic demeurent sensiblement les mêmes. mais définissons d'abord la manifestation qui désigne l'ensemble des troubles. Les tics consistent en un mouvement ou une vocalisation qui survient de manière soudaine, rapide, récurrente et non rythmée. La notion de stéréotypie, présente dans la description du DSM-IV-TR, a été retirée. Les tics moteurs et sonores peuvent être déterminés comme étant simples ou complexes. Par exemple, un tic moteur simple est bref, comme un clignement excessif des yeux, alors qu'un tic moteur complexe peut regrouper plusieurs tics moteurs simples ou plus d'un groupe de muscles, tel qu'un spasme de l'abdomen. Le Tableau 1 présente quelques exemples de tics moteurs et sonores.

Il est possible de concevoir un continuum de gravité entre les différents troubles tic, en considérant principalement la durée et le type de manifestations. Les tics spécifiés/non spécifiés se distinguent par le fait que le diagnostic ne rencontre pas les critères pour l'un des autres troubles tics ou parce que l'information est insuffisante pour établir un diagnostic plus précis. Les tics provisoires impliquent la manifestation de tics moteurs et/ou sonores, durant moins d'un an. Le critère de durée est maintenant plus flexible que dans le DSM-IV-TR qui précisait que les tics devaient être présents presque tous les jours, pendant au moins quatre semaines mais pas plus de douze mois. Dans le trouble tic persistant ou chronique, les tics manifestés doivent être moteurs ou sonores et ils doivent être présents depuis plus d'un an. Enfin, le syndrome de Gilles de la Tourette (SGT) se caractérise par la présence de plusieurs tics moteurs et d'au moins un tic sonore au cours de son évolution. Les tics doivent persister au moins un an après l'apparition de la première manifestation. Le DSM-IV-TR excluait le diagnostic de SGT si les tics s'interrompaient durant trois mois, mais ce critère n'apparait pas dans le DSM-5 en raison de la fluctuation naturelle de la fréquence des tics. Pour tous les troubles tic, les symptômes ne doivent pas être causés par les effets 
Tableau 1

Exemples de tics moteurs et sonores

\begin{tabular}{lll}
\hline Tics & Moteur & Sonore \\
\hline Simple & Cligner/rouler des yeux & Renifler - Racler la gorge \\
& Hausser une épaule & Bruits de bouche \\
& Secouer la tête & Claquer la langue \\
& Plisser le nez & Éructer \\
& Froncer les sourcils & Gémir/petits cris \\
& & \\
Complexe & Sautiller & Écholalie - Palilalie \\
& Toucher/pianoter & Respiration rythmée \\
& Spasme de l'abdomen & Séquence de sons \\
& Plier/étirer la jambe & Fredonner \\
& Échokinésie & Coprolalie \\
\hline
\end{tabular}

physiologiques d'une substance ou par une autre maladie. Par ailleurs, le critère indiquant qu'une souffrance marquée ou que l'altération significative du fonctionnement est nécessaire au diagnostic a été retiré, bien que cet aspect clinique soit abordé dans la description des troubles tic du DSM-5.

\section{LE SYNDROME DE GILLES DE LA TOURETTE}

Le SGT est un trouble neurodéveloppemental dont la prévalence est actuellement estimée entre $0,1 \%$ et $1 \%$ en population générale (Scharf, Miller, Mathews, \& Ben-Shlomo, 2012). Cette prévalence varie toutefois selon les caractéristiques de la population étudiée, les critères diagnostiques et les méthodes d'évaluation utilisées. En effet, l'expression variable du syndrome peut faire en sorte que les formes les moins sévères soient moins reconnues et diagnostiquées (O'Connor, 2002).

Les tics fluctuent en fréquence, en intensité et en localisation selon chaque personne et en fonction de l'évolution du syndrome (APA, 2013). La variabilité des tics correspond généralement à un patron individuel où les tics se manifestent plus ou moins en fonction de l'activité en cours. Par exemple, les tics peuvent être plus fréquents pour quelqu'un lorsqu'il est plus stressé, tandis qu'une autre personne peut manifester davantage de tics lorsqu'elle est détendue ou concentrée (p. ex., devant la télévision ou en travaillant). Le profil individuel semble dépendre d'un état physiologique lié à la situation et à ce qu'elle représente pour la personne (O'Connor, 2005). Par ailleurs, il est important de faire la distinction entre des stratégies d'autocontrôle apprises en contexte thérapeutique (ou par la personne elle-même), permettant de diminuer la fréquence des tics, et le comportement de retenir ses tics. Certaines personnes peuvent réprimer leurs tics durant un certain temps (p. ex., à l'école ou en réunion), mais la rétention des manifestations n'a pas le même effet que l'application d'une stratégie d'autocontrôle; elle induit une tension musculaire et globale qui 
peut engendrer des maux de tête ou un sentiment global de frustration (Himle \& Woods, 2005; Meidinger, Miltenberger, Himle, Omvig, Trainor, \& Crosby, 2005).

L'âge moyen du diagnostic de SGT est de 6 ans et le diagnostic est de deux à quatre fois plus fréquent chez les garçons que chez les filles (APA, 2013). Les premiers symptômes sont généralement des tics moteurs simples, qui surviennent dans la partie supérieure du corps. Les tics sonores et les tics complexes se développent en moyenne vers l'âge de 11 et 12 ans. Les symptômes semblent atteindre leur sévérité maximale au début de l'adolescence et ils tendent à s'améliorer ou à demeurer stable à l'âge adulte (APA, 2013; Robertson, 2000).

\section{Étiologie du SGT}

Les théories explicatives du SGT ont évolué au fil des ans, mais l'étiologie du trouble demeure encore aujourd'hui incertaine. Le trouble semble néanmoins présenter une forte composante biologique et génétique. Dans une revue de la littérature scientifique, Paschou (2013) identifie l'implication des récepteurs DRD2 de la dopamine et des récepteurs MAO-A (monoamine oxydase $A$ ) des systèmes dopaminergique et sérotoninergique, quoique les résultats à ce sujet demeurent inconsistants. L'étude des déterminants génétiques du SGT suggère l'implication de plusieurs gènes, mais des mutations dans le gène SLITRK1 (SLIT-NTRK-like family, member 1) ont été identifiées chez un certain nombre de patients atteints du SGT. La contribution génétique la plus importante à l'expression du SGT semble toutefois pointer vers l'expression de gènes impliqués dans les voies histaminergiques et glutamatergiques (Paschou, 2013). Évidemment, des facteurs environnementaux ou épigénétiques contribuent à l'apparition ou à l'expression du syndrome. Une recension des écrits révèlent que les évènements prénataux (tabagisme maternel et stress), périnataux (faible poids à la naissance et utilisation de forceps) et les stades de la vie postnatale (réactions immunitaires) peuvent contribuer à l'apparition et à l'évolution du SGT (Hoekstra, Dietrich, Edwards, Elamin, \&, Martino, 2013). Ainsi, l'interaction complexe entre des facteurs génétiques et environnementaux semble responsable de l'origine du SGT et de la variabilité de ses manifestations.

Comme le SGT est souvent associé à d'autres troubles psychiatriques, les profils neurologiques des individus qui en sont atteints couvrent un large spectre d'expression. Toutefois, des caractéristiques neurobiologiques spécifiques sont présentées dans la littérature scientifique. D'abord, tel que mentionné précédemment, le SGT est associé au débalancement de certains neurotransmetteurs, dont la dopamine qui régit l'activité motrice et qui serait liée aux tics 
caractéristiques du syndrome. Une hyperactivité des systèmes dopaminergiques semble entrainer une désinhibition comportementale et une hyperactivité motrice (Hallet, 2015; Jackson, Draper, Dyke, Pépés, \& Jackson, 2015; Serajee \& Huq, 2015). Le système dopaminergique est également impliqué dans le système de la récompense de telle sorte qu'il a été suggéré que les tics représentent une manifestation visant à soulager un certain désir ou une envie (" urge »), poussant ainsi l'individu à reproduire le comportement qui devient une habitude sous le contrôle d'un renforcement négatif (Hallett, 2015; Jackson et al., 2015). Les principaux neurotransmetteurs qui seraient associés à ce phénomène sont la dopamine (DA), fortement reliée aux mécanismes de renforcement des apprentissages, et le GABA, principal inhibiteur du système nerveux central. Ensemble, ces dysfonctionnements de DA et GABA peuvent contribuer à un dysfonctionnement des circuits cortico-striato-thalamiquecortical (CSTC) impliqués dans l'apprentissage moteur, en particulier la formation des habitudes, et du choix des actions selon le contexte comportemental (Jackson et al., 2015). Des anomalies structurelles ou fonctionnelles dans les boucles du circuit CSTC interfèrent également avec la mise en œuvre des actions volontaires, suggérant une relation entre les changements dans ces structures et les tics (Vicario, Gulisano, Martino, \& Rizzo, 2015). Wang et ses collaborateurs (2014) suggèrent que les tics seraient causés par les effets combinés de l'activité excessive dans les voies motrices et de l'activation réduite dans les portions de contrôle des circuits CSTC. Par ailleurs, un modèle conceptuel du SGT, développé par O'Connor (2002), propose que la survenue des tics résulte de l'interaction entre des processus physiologiques de base et des facteurs cognitifs. La prémisse est donc que les tics visent à libérer une tension musculaire, secondaire à un déclencheur situationnel. Autrement dit, les individus ayant le SGT présenteraient un style d'action particulier, caractérisé par de la surpréparation et de la suractivité, qui tend à augmenter l'activité sensorimotrice et la tension musculaire de la personne qui, finalement, déclenche le tic qui a une fonction d'autorégulation.

Il semble que les individus atteints du SGT présentent une dysfonction au niveau des lobes frontaux, associés à l'inhibition des comportements, la planification motrice et l'autocontrôle. Des changements structuraux sont aussi observés dans les réseaux sensorimoteurs. Notamment, un amincissement du cortex sensori-moteur, de même qu'une diminution des connexions de ce cortex avec les ganglions de la base et le thalamus (Worbe, Lehericy, \& Hartmann, 2015) ont été notés de même qu'une légère suractivation de cervelet et du cortex prémoteur au repos (Jankovic \& Kurlan, 2011). Une réduction du volume des noyaux caudés a été observée (Jackson et al., 2015), ces derniers étant d'ailleurs directement impliqués dans les circuits CSTC. Chez l'enfant, la présence d'une hypertrophie du cortex limbique préfrontal et une corrélation entre la taille 
du corps calleux et l'occurrence des symptômes sont suggérées (Jankovic \& Kurlan, 2011; Serajee \& Huq, 2015).

Les changements neurologiques fonctionnels et structurels chez les personnes ayant le SGT peuvent être conçus comme des causes premières du trouble, tout comme des conséquences associées aux symptômes cliniques; ils doivent donc être interprétés avec prudence (Jackson et al., 2015). En outre, la neuro-imagerie, l'électrophysiologie et les tests biochimiques et génétiques moléculaires ne suffisent pas pour confirmer le diagnostic.

\section{Profil cognitif du SGT}

Cliniquement, les anomalies neurologiques tout justes citées semblent engendrer des conséquences plus larges que les tics, qui se traduisent par des atteintes dans le fonctionnement cognitif. Les avancées en recherche indiquent présentement des profils cognitifs variables d'un individu à l'autre. L'évaluation neuropsychologique auprès des jeunes ayant le SGT suggère un niveau d'aptitude équivalent à celui des enfants neurotypiques (Como, 2001). Toutefois, Debes et al. ont observé, chez un groupe de 266 enfants ayant le SGT, un quotient intellectuel légèrement plus faible (moins d'un écart-type) que celui de la population générale et d'un groupe contrôle, spécifiant que l'apparition précoce des tics et la présence d'un trouble associé semblent liées à ce résultat (Debes, Lange, Jessen, Hjalgrim, \& Skov, 2011). D'autres études ont révélé la présence de déficits au niveau de la motricité fine, de l'intégration visuomotrice, de l'attention visuospatiale et des fonctions exécutives comme la planification de l'action, la flexibilité cognitive, le contrôle de l'inhibition, la résolution de problèmes et le jugement abstrait, de la fluidité verbale et de la mémoire non verbale (Cavanna, Eddy, \& Rickards, 2009; Como, 2001; Eddy, Rizzo, \& Cavanna, 2009; Lavoie, Leclerc, \& O'Connor, 2013). Sur le plan scolaire et académique, la prévalence des troubles d'apprentissage dans le SGT serait équivalente à celle de la population générale, mais certaines données suggèrent des difficultés spécifiques en mathématiques et en langage écrit (Como, 2001). Les études révèlent toutefois peu de constance dans les résultats, étant donné la difficulté à déterminer ce qui découle directement du SGT et non des manifestations des troubles concomitants. Des études plus conservatrices ont été effectuées sur des personnes ayant le SGT "sans complication». Les résultats suggèrent que la seule évidence robuste d'altération significative se manifesterait dans la performance des sujets à certaines tâches d'inhibition (Eddy et al., 2009). Plus récemment, une recension des écrits concernant la cognition sociale chez les adultes ayant le SGT montre une altération subtile de la cognition sociale, notamment lors d'épreuves nécessitant l'intégration d'informations émotionnelles, de perspectives conflictuelles et une prise de décision. Ce qui serait, selon les auteurs, conséquente à une réponse non 
conventionnelle aux stimuli sociaux plutôt qu'à une théorie de l'esprit défaillante (Eddy \& Cavana, 2013).

\section{Troubles associés}

L'absence de troubles associés au SGT est plutôt rare et serait propre à seulement $10 \%$ de cette population (Ganos \& Martino, 2015). Le SGT est, de manière générale, compliqué par des troubles psychiatriques ou psychologiques concomitants, dont les conséquences interfèrent plus souvent avec la vie quotidienne que l'expression des tics en soi (Lavoie et al., 2013). De plus, le pronostic est généralement moins favorable en présence de plus d'un trouble concomitant (Freeman, Fast, Burd, Kerbeshian, \& Sandor, 2000).

Le trouble déficitaire de l'attention avec hyperactivité (TDAH) et le trouble obsessionnel-compulsif (TOC) sont fréquemment associés au SGT et leur présence serait associée à une augmentation des déficits cognitifs et des troubles d'apprentissage (Como, 2001). Une vaste étude internationale a rapporté que le TDAH est présent chez environ $55 \%$ des enfants atteints du SGT et qu'il représente ainsi le principal trouble associé au SGT (Freeman \& Tourette Syndrome International Database Consortium, 2007). Le TDAH se caractérise par la présence de symptômes d'inattention, d'hyperactivité et d'impulsivité qui interfèrent avec le fonctionnement général de la personne (APA, 2013). Typiquement, l'apparition du TDAH précède l'apparition des tics. De plus, les symptômes extériorisés conséquents à ces deux troubles sont plus élevés en fréquence et en intensité lorsqu'ils apparaissent de manière simultanée chez un même individu. Conséquemment, les capacités d'adaptation psychosociale s'en voient souvent diminuées (Leclerc, Forget, \& O'Connor, 2008). Puisque les symptômes de ces troubles neurodéveloppementaux se chevauchent, il peut être difficile de les différencier cliniquement. Selon la recension des écrits de Noton-Durand et Jalenques (2008), on observe chez ces jeunes de l'impulsivité, une hyperactivité motrice, des difficultés d'attention et de concentration ainsi qu'une désobéissance marquée. Un léger déficit quant au traitement complexe des émotions, particulièrement avec les items impliquant la colère a également été observé chez l'enfant ayant le SGT+TDAH, ainsi que chez l'adulte ayant le SGT seul (Druy, Channon, Barrett, Young, Stern, Simmons, \& Crawford, 2012). De plus, les difficultés scolaires et relationnelles peuvent s'avérer importantes dans cette population et près de la moitié d'entre eux auraient recours à une éducation spécialisée. Le déficit des fonctions exécutives est plus prononcé lorsque les troubles apparaissent simultanément et seraient intimement lié aux dysfonctions des circuits fronto-striatal et fronto-pariétal (Coffey, 2015). La suractivation des neurotransmetteurs DA dans le système nerveux central, tout comme l'inhibition motrice déficitaire, serait à la fois propre aux tics ainsi qu'à 
I'hyperactivité, laissant présager certaines similitudes dans l'étiologie de ces troubles (Coffey, 2015; Lebowitz et al., 2012). Certains auteurs estiment même que le SGT, le TDAH et le TOC seraient l'expression différentielle d'un même processus étiopathogénique (Fourneret, Desombre \& Broussolle, 2014). Enfin, bien que le TDAH ne semble pas corrélé avec la sévérité des tics, il serait intimement lié à la présence de comportements agressifs et turbulents dans le SGT. D'ailleurs, la présence de TDAH concomitant augmente la probabilité de développer un trouble extériorisé additionnel tels que le trouble oppositionnel avec provocation (Lebowitz et al., 2012) et les épisodes explosifs (Budman, Rockmore, Stokes, \& Sossin, 2003).

Le TOC est présent dans approximativement $30 \%$ à $50 \%$ de la population souffrant du SGT. II apparaît toutefois plus tardivement dans le développement, généralement après l'apparition des tics (Thibault, Felezeu, O'Connor, Todorov, Stip, \& Lavoie, 2008). Le TOC se caractérise par la présence d'obsessions ou de compulsions dont la personne reconnait la nature excessive ou déraisonnable, sans toutefois avoir de contrôle sur elles (Chamberlain, Blackwell, Fineberg, Robbins, \& Sahakian, 2005). L'aspect répétitif lié aux TOC peut s'apparenter aux tics présents dans le SGT d'un point de vue comportemental et la distinction entre les troubles peut s'avérer ardue. C'est la fonction des manifestations qui les distinguent puisque les tics, pouvant être confondus avec les compulsions du TOC, ne visent pourtant pas à soulager ou à neutraliser l'anxiété (Fourneret et al., 2014). Le besoin de symétrie ou de toucher des objets d'une manière donnée est typique aux personnes ayant le SGT et semble répondre davantage à un besoin physiologique (Leclerc et al., 2008). Par ailleurs, la sévérité des tics serait significativement plus importante chez les individus présentant les deux troubles simultanément (Lebowitz et al., 2012). Comme le SGT, le TOC semble provoqué par un défaut des fonctions inhibitrices, reliées à la réduction du volume des ganglions de la base et de ses projections dans les aires frontales (dans le cortex moteur primaire pour le SGT et dans le cortex cingulaire orbitofrontal antérieur latéral pour le TOC) (Thibault et al., 2008). Les personnes présentant un TOC associé au SGT semblent également montrer des dysfonctions dans le circuit orbitofrontal (Eddy et al., 2009). Sur le plan neuropsychologique, une étude suggère que l'association SGT et TOC n'affecte pas l'attention ni la mémoire, mais plutôt, les fonctions exécutives sur le plan de l'inhibition de la réponse et de l'autorégulation comportementale (Müller et al., 2003). Toutefois, ces résultats n'ont pas été comparés à ceux d'un groupe contrôle (SGT sans TOC). Par ailleurs, une autre étude suggère que le profil cognitif serait lié aux symptômes obsessionnel-compulsifs (SOC) plutôt qu'au TOC en général, observant une rigidité cognitive plus importante chez les participants ayant le SGT et présentant la dimension de symptômes d'agression que chez ceux ne présentant pas ces 
symptômes, alors que les participants avec ou sans besoin de symétrie ne présentaient pas de différence (Matsuda et al., 2012). Finalement, la probabilité de développer un autre trouble internalisé comme la dépression ou un autre trouble anxieux est plus grande au sein des personnes ayant le SGT + SOC (Fourneret et al., 2014), chez lesquels on rapporte d'ailleurs certains comportements d'automutilation (Thibault et al., 2008).

Des recensions d'écrits ont souligné d'autres troubles psychologiques associés au SGT (Hamel, O'Connor, Morand-Beaulieu, \& Lavoie, 2015; Leclerc et al., 2008). Par exemple, les troubles d'apprentissage touchent près du tiers des individus ayant le SGT et $60 \%$ d'entre eux ont des difficultés affectant le rendement scolaire. On retrouve aussi des perturbations du sommeil (p. ex., insomnie, somnambulisme et mouvements périodiques des jambes), des migraines, ainsi que des troubles liés à l'intégration visuelle et motrice (p. ex., dyspraxie). La prévalence des troubles de l'humeur et des troubles anxieux semble plus importante dans cette population clinique que dans la population générale. Le contrôle de la colère et des impulsions est particulièrement affecté chez les jeunes atteints du SGT, ce qui engendre souvent une détresse chez ces derniers, ainsi que chez leurs proches. La colère, l'opposition et les comportements agressifs sont présents chez environ $50 \%$ des enfants ayant le SGT (Alsobrook \& Pauls, 2002). Les épisodes explosifs, décrits comme des crises violentes qui se produisent d'une manière soudaine et récurrente, se distinguent de la colère par leur intensité disproportionnée par rapport au déclencheur (Budman et al., 2003). Ils semblent résulter de l'interaction entre les différents symptômes de troubles associés; l'impulsivité et le manque de contrôle de soi liés au TDAH, en plus du perfectionnisme et de la rigidité cognitive liés aux TOC, peuvent entraîner un mélange explosif que les parents considèrent souvent comme les symptômes qui interfèrent le plus dans la famille ainsi que dans le domaine scolaire (Sukhodolsky et al., 2003). Finalement, la proportion élevée de concomitance avec certaines problématiques soulève la possibilité que ces dernières fassent partie intégrante du syndrome et qu'elles constituent son profil clinique.

\section{Évaluation diagnostique}

L'évaluation diagnostique du SGT s'effectue en fonction d'observations clinique; ce sont l'histoire médicale et comportementale, ainsi que la présence des symptômes qui prévalent. Les instruments de mesure utilisés pour l'évaluation diagnostique du SGT visent tous à documenter les manifestations, principalement les tics, en termes de présence, de durée, de fréquence et d'intensité. Les questionnaires peuvent être complétés sous forme d'entrevue semi-structurée à l'aide d'un professionnel ou par auto-notation. Dans tous les cas, l'observation directe 
ou indirecte (p. ex., enregistrement vidéo) bonifie grandement le processus diagnostic.

Deux instruments de mesure sont principalement utilisés en milieu clinique et dans les études portant sur le SGT. Ces deux questionnaires se basent sur une liste de chaque partie du corps et sur les tics qui y sont généralement associés, et ce, en fonction de la dimension des tics (moteur, sonore, complexité).

Le Yale Global Tic Severity Scale (YGTSS; Leckman et al., 1989) est l'un des instruments de mesure les plus utilisés en recherche (Abramsvitch et al., 2015). Le YGTSS évalue cinq dimensions des tics (le nombre, la fréquence, l'intensité, la complexité et l'interférence), ainsi que le degré d'altération du fonctionnement quotidien de la personne. La fidélité interjuges varie de $r=0,52$ à 0,99 selon les dimensions et la validité factorielle représente $8 \%$ de la variance expliquée à l'aide de deux dimensions, l'une pour les tics moteurs et l'altération du fonctionnement et l'autre pour les tics sonores (Leckman et al., 1989).

Le Tourette's Syndrome Global Scale (TSGS; Harcherik, Leckman, Detlor. \& Cohen, 1984) est une mesure multidimensionnelle de la sévérité (interférence) et de la fréquence des tics moteurs et sonores (simples et complexes). Le fonctionnement social de la personne est aussi évalué en fonction de la conduite, de l'agitation motrice et des comportements à l'école ou au travail). Cet instrument possède de bonnes propriétés psychométriques avec des coefficients de cohérence interne $(\alpha=0,89)$, de fidélité test-retest $(r=0,46$ à $r=0,99)$ et d'accord interjuges $(r=0,65$ et $r=0,85)$ satisfaisants.

D'autres questionnaires se basent sur l'auto-évaluation des tics chez les adultes ou sur l'évaluation des parents d'un jeune ayant des tics. II est possible de citer en exemple le Motor tic, Obsessions and compulsions, Vocal tic Evaluation Survey (MOVES; Gaffney, Sieg, \& Hellings, 1994), le Tourette's Disorder Scale (Shytle et al., 2003), Parent Tic Questionnaire (PTQ) (Chang, Himle, Tucker, Woods, \& Piacentini, 2009), le Child Tourette's Disorder Impairment Scale-Parent Version (Storch, Lack, Simons, Goodman, Murphy, \& Geffken, 2007) et le Adult Tic Questionnaire (ATQ) (Abramovitch et al., 2015). L'ensemble des questionnaires présentés n'ont toutefois pas de traductions francophones validées et il est parfois difficile de se procurer certains d'entre eux pour l'évaluation clinique.

\section{Évaluation neuropsychologique}

Puisque le SGT est souvent associé à un dysfonctionnement exécutif, plusieurs études ont tenté d'évaluer la performance d'individus qui en sont 
atteints à partir de tests d'attention, de perception, de mémoire et d'inhibition motrice, entre autres. Eddy et ses collaborateurs (2009) ont recensé les résultats de plusieurs études ayant pour objectif de dresser le profil neuropsychologique et des fonctions exécutives dans le SGT. La compilation des résultats montre que le large spectre d'expression du SGT et l'effet confondant possible de concomitances, qui ne sont pas toujours contrôlées dans les devis d'études, font en sorte que les résultats manquent de constance. Par conséquent, il existe un réel défi dans l'application de tests neuropsychologiques visant à l'évaluation des déficits cognitifs et à l'établissement de diagnostic différentiel au SGT. Le même constat apparaît dans d'autres études, comme celle de Lavoie et ses collaborateurs (2007), visant à discriminer le profil neuropsychologique du SGT de celui du trouble tic chronique, ou la recension des écrits de Hamel et ses collaborateurs (2015) qui présente un état des connaissances concernant l'impulsivité dans le SGT et qui propose un lien entre les comportements impulsifs, la flexibilité cognitive et l'inhibition, éléments importants du système exécutif. Malgré tout, plusieurs tests neuropsychologiques sont utilisés pour évaluer les fonctions exécutives et permettre de dresser un portrait des fonctions cognitives des personnes ayant le SGT. Quelques-uns des principaux tests utilisés à ces fins sont présentés ci-dessous.

Le test de la figure complexe de Rey-Osterrieth peut être utilisé pour évaluer l'attention visuelle, les capacités visuo-spatiales, l'organisation et la mémoire (Lavoie, 2007). La figure complexe de Rey sollicite particulièrement le cortex postéro-temporo-pariétal soulignant l'implication de la cognition visuo-spatiale dans la construction de la figure. Les études montrent que les enfants SGT ayant un TDAH concomitant éprouvent des difficultés dans ce test alors que les résultats varient en l'absence de TDAH (Eddy et al., 2009). Ensuite, le test de Hayling est un test verbal constitué de deux sections de 15 phrases dans chacune desquelles un mot est manquant. Associé au cortex angulaire supérieur (inhibition, processus intentionnels, attention), ce test a été utilisé pour évaluer l'inhibition de la réponse verbale dans le SGT, qui serait déficitaire même dans les cas de SGT sans concomitance (Eddy et al., 2009; Hamel et al., 2015). Tel que relevé dans la recension des écrits de Hamel et collaborateurs (2015), le test de Stroop existe sous différentes variantes et vise à évaluer l'attention sélective ou les capacités d'inhibition, mais la performance des individus ayant le SGT est variable. Le test de Flanker évalue l'inhibition de la réponse en utilisant trois types de stimuli: congruents, incongruents et neutres. Des performances atypiques ont été rapportés auprès de personnes ayant le SGT (Crawford 2005), ils mais n'ont pas été répliqués par la suite (Channon, 2006). L'épreuve «Go no Go » (GNG) est un test d'inhibition motrice non verbale défini par deux classes de stimuli. L'une nécessite l'exécution d'une réponse motrice 
(« go »). Par opposition, les stimuli distracteurs demandent l'inhibition de ce comportement («no go »). Ces épreuves évaluent principalement la capacité à contenir les réponses aux stimuli distracteurs. Déjà utilisés aurpès d'individus ayant le SGT, la plupart des résultats aux études rapportées par Eddy et ses collaborateurs (2009) ou Hamel et ses collaborateurs (2015) n'ont pas été en mesure d'observer une différence significative dans les performances aux tests GNG chez des sujets ayant le SGT sans comorbidité, comparativement à des sujets contrôles. Très peu d'études concernent l'évaluation de la cognition sociale, mais le test des Faux pas serait particulièrement sensible aux altérations de la cognition sociale chez l'adulte ayant le SGT (Eddy \& Cavana, 2013). L'Index de réactivité émotionnelle (IRE) a également été utilisé par Eddy et ses collaborateurs (2015) afin d'évaluer l'empathie et la tendance à adopter la perspective d'autrui dans le SGT. Les chercheurs ont mis en parallèle les résultats à l'IRE avec la gravité des tics et la présence de troubles associés tels que l'anxiété, la dépression, le TOC et le TDAH. Les résultats montrent que le SGT serait associé à des changements dans la cognition sociale, sans distinction en fonction de la concomitance. Toutefois, la sévérité des tics serait inversement proportionnelle à la détresse rapportée. La flexibilité cognitive peut être évaluée par des tests neuropsychologiques, notamment le Wisconsin Card Sorting Test (WCST). Des études recensées par Eddy et ses collaborateurs (2009), ainsi que l'étude de Lavoie et collaborateurs (2007) montrent une performance intacte à ce test par des individus ayant un SGT avec ou sans comorbidité. Toutefois, une étude plus récente indique que les résultats à ce test permettent de distinguer les participants ayant un SGT des participants d'un groupe contrôle, les auteurs allant jusqu'à considérer les déficits retrouvés dans le WCST comme un endophénotype neurocognitif du SGT (Eddy \& Cavanna, 2014).

En somme, l'interprétation des résultats aux tests neuropsychologiques auprès de personnes ayant le SGT demeure un défi puisqu'ils ne permettent pas d'établir clairement un profil des fonctions cognitives et exécutives complètement distinc, en raions de la présence fréquente de troubles associés. D'autres études sont donc nécessaires pour mieux distinguer les différents phénotypes.

\section{INTERVENTIONS PRÉCONISÉES}

Bien que les tics soient considérés comme la manifestation comportementale d'un trouble neurodéveloppemental, il est impératif de déterminer les meilleures pratiques thérapeutiques permettant de mieux gérer les tics par les personnes qui en sont atteintes, grâce à l'apprentissage de stratégies d'autocontrôle. Ainsi, les lignes directrices liées à la pratique canadienne, européenne et américaine recommandent 
toutes une combinaison de la médication et d'un traitement cognitifcomportemental pour la diminution de la fréquence et de l'intensité des tics (Murphy, Lewin, Storch, Stock, \& the American Academy of Child and Adolescent Psychiatry Committee on Quality Issues, 2013; Pringsheim et al., 2012; Verdellen, van de Griendt, Hartmann, Murphy, \& ESSTS Guidelines Group, 2011).

\section{Pharmacothérapie}

La pharmacologie permet de diminuer les tics dans de nombreux cas, malgré les effets secondaires potentiels (Eddy et al., 2011; Scahill et al., 2006; Steeves et al., 2012). Les effets chez les enfants et les adolescents varient entre $35 \%$ et $90 \%$ (Copur, Arpaci, Demir, \& Narin, 2007; Ghanizadeh \& Haghighi, 2014; Lyon et al., 2009; Yoo et al., 2011). La classe de médication la plus souvent utilisée auprès des jeunes est celle des antipsychotiques (p. ex., Aripripazole, Quetiapine, Risperidone, Haloperidol) (Dion, Annable, Sandor, \& Chouinard, 2002; Freeman et al., 2000; Gilbert, Batterson, Sethuraman, \& Sallee, 2004; Roessner, Schoenefeld, Buse, Bender, Ehrlich, \& Münchau, 2013). D'autres classes de médication sont aussi représentées dans la population clinique (p. ex., antihypertenseur, psychostimulant, antidépresseur, anxiolytique), en fonction des symptômes dominant le portrait clinique (Freeman et al., 2000; Roessner et al., 2013). Plusieurs études évaluant l'effet de la pharmacothérapie consistent en des études de cas, ce qui limite la généralisation des résultats. Par ailleurs, les effets de l'utilisation à long terme de certaines molécules sont à considérer chez les enfants et les adolescents (Eddy et al., 2011; Roessner et al., 2013).

\section{Psychothérapie}

Un suivi thérapeutique permet à la personne atteinte du SGT, ainsi qu'à sa famille et à ses proches, de mieux comprendre les symptômes et les conséquences du syndrome qui compromettent le développement. Une intervention visant le soutien émotif et l'éducation psychologique permettant de pallier notamment à une faible estime de soi, à la détresse face aux symptômes, à un épisode dépressif ou à un manque d'habiletés sociales (Leclerc et al., 2008).

\section{Thérapie cognitive et comportementale}

Les thérapies cognitives et comportementales (TCC) permettent de modifier des comportements cibles tout en procédant à une rééducation des pensées entourant ces comportements. Les interventions préconisées dans cette approche visent donc l'enseignement de stratégies d'autocontrôle (Frank \& Cavanna, 2012). 
Le renversement d'habitude $(\mathrm{RH})$ constitue le premier traitement comportemental efficace et reconnu pour la diminution des tics (Azrin \& Nunn, 1973). Le modèle d'intervention, basé principalement sur les théories de l'apprentissage, se fonde sur l'hypothèse qu'à l'origine le tic est une réaction normale, accentuée en termes de fréquence et maintenue par un processus de conditionnement. Le RH comporte cinq étapes thérapeutiques (Azrin \& Peterson, 1988) : 1) entraînement à la prise de conscience ( $p$. ex., description de la séquence comportementale, autonotation); 2) entraînement à la relaxation musculaire progressive (Jacobson, E. : Progressive relaxation, $2^{\mathrm{e}}$ édition, 1938); 3 ) entraînement à une réponse incompatible ( $p$. ex., contraction graduée des muscles antagonistes de manière à empêcher le tic de se manifester); 4) gestion de la contingence (p. ex., soutien social et renforcement positif); 5) généralisation des apprentissages ( $p$. ex., pratiquer la réponse incompatible dans différents contextes pour augmenter la probabilité qu'elle soit émise spontanément). Les composantes déterminantes du RH sont l'entraînement à la prise de conscience et l'entraînement à une réponse incompatible (Clarke, Bray, Kehle, \& Truscott 2001; Dufrene, Lestremau Harpole, Sterling, Perry, Burton, \& Zoder-Martell, 2013). Les études portant sur l'effet du RH montrent une diminution de la fréquence des tics de $40 \%$ à $100 \%$ avec un maintien des améliorations jusqu'à deux ans plus tard (Azrin \& Peterson, 1988; Clarke et al., 2001; Deckersbach et al., 2006; Watson, Howell, \& Smith, 2001; Wilhelm, Deckersbach, Coffey, Bohne, Peterson, \& Baer 2003; Woods \& Miltenberger, 1995).

Le Comprehensive Behavioral Intervention for Tics (CBIT) (Woods, Piacentini, Chang, Deckersbach, Ginsburg, \& Peterson, 2008) consiste en une révision du $\mathrm{RH}$ puisqu'il inclut les étapes d'entraînement à la prise de conscience, à une réponse incompatible et à la relaxation musculaire, mais le CBIT souligne l'importance des antécédents et des conséquences aux tics qui peuvent influencer la probabilité de manifester des tics selon un processus de conditionnement opérant (Capriotti, Espil, Conelea, \& Woods, 2013; Himle et al., 2014). Les interactions entre l'individu et son environnement sont responsables de la variabilité de l'expression des tics; le modèle considère les variables biologiques, environnementales et comportementales (p. ex., stress, anxiété, frustration, faire rire des tics, attention) (Woods et al., 2008). Ainsi, des procédures d'éducation psychologique et d'intervention fonctionnelle sont ajoutées aux composantes thérapeutiques du RH pour compléter le CBIT (Woods et al., 2008). Ce traitement montre de bons résultats auprès des enfants et des adultes manifestant des tics (Hwang, Tillberg, \& Scahill, 2012; Piacentini et al., 2010; Woods et al., 2011) et son utilisation est généralement recommandée (Pringsheim et al., 2012). 
Thérapie cognitive, comportementale et psychophysiologique

Deux modèles d'intervention ciblent principalement la dynamique physiologique qui sous-tend les manifestations de tics. Le premier traitement se base sur le principe que les tics se déclenchent et se maintiennent par un processus de renforcement négatif où le tic serait une réponse comportementale permettant la neutralisation d'une sensation désagréable et la réduction de la tension musculaire et de l'activation sensori-motrice (Hoogduin, Verdellen, \& Cath, 1997). Le traitement consiste donc en un processus d'exposition avec prévention de la réponse (EPR) visant à interrompre cette séquence (Evers \& van de Wetering, 1994; Hoogduin et al., 1997; Verdellen, Keijsers, Cath, \& Hoogduin, 2004). La personne doit d'abord identifier la sensation dite prémonitoire (ou tic sensoriel) puis, résister à la manifestation du tic par un entraînement à tolérer la sensation et les signes avant-coureurs. Ainsi, la personne apprend à résister à l'émission des tics tout en ressentant pleinement les sensations associées, afin de mettre fin aux sensations physiques précédant l'émission de tics considérées comme désagréables (Hoogduin et al., 1997). L'EPR montre des effets intéressants, mais le traitement exige une tolérance à la détresse de la part de la personne ayant des tics et par conséquent, cette intervention ne peut être recommandée pour tout le monde (Pringsheim et al., 2012; Verdellen et al., 2011).

Les traitements décrits précédemment ciblent le tic de manière précise, mais les tics sont reconnus pour leur fluctuation en termes de fréquence, d'intensité et de localisation (APA, 2013). Ainsi, un modèle thérapeutique global favorisant un travail sur les processus sous-jacents aux manifestations de tics semble intéressant et plus approprié à la nature complexe d'un trouble neurodéveloppemental. Le modèle cognitif et psychophysiologique de O'Connor $(2002,2005)$ met en évidence l'interaction de facteurs cognitifs, comportementaux et psychophysiologiques dans le déclenchement et le maintien des tics. Des études ont montré que les adultes manifestant des tics auraient des styles de planification de l'action (p. ex., la manière dont l'individu agit, organise et planifie ses activités) qui tendent à augmenter l'activation sensorimotrice et la tension musculaire (O'Connor, Lavoie, Blanchet, \& St-PierreDelorme, 2015; O'Connor, Lavoie, Stip, Borgeat, \& Laverdure, 2008). La conséquence psychophysiologique de ces styles d'action est l'augmentation de l'activation sensori-motrice et de la tension musculaire. Ainsi, les tics auraient une fonction d'autorégulation sensori-motrice; ils permettent de relâcher la tension musculaire accumulée, mais ce qui à long terme renforce le cycle maintenant les tics. Le traitement découlant de cette approche, le Cognitive and psychophysiological treatment (CoPs), vise la prévention de l'émergence du tic par une restructuration globale du comportement et par une rééducation de la pensée liée à la planification du mouvement (O'Connor, 2005). Le traitement a été adapté pour les 
enfants manifestant des tics, cette version de la thérapie se nomme Façotik (Leclerc, Goulet, Hamel, \& O'Connor, 2013). Les deux thérapies sont structurées avec guides méthodologiques et elles abordent dix thèmes thérapeutiques (voir Tableau 2). Les études montrent de très bons résultats quant à la diminution des tics après l'intervention (30\% à $75 \%$ ) chez les adultes (O'Connor, Brault, Loiselle, Robillard, Borgeat, \& Stip, 2001; O'Connor, Laverdure, Taillon, Stip, Borgeat, Lavoie, 2009; O'Connor et al., 2015) et chez les enfants (Leclerc \& O'Connor, 2015; Leclerc, Valois, J-Nolin, Bombardier, Ouellette, \& O'Connor, en préparation).

\section{CONCLUSION}

Les premières études systématiques concernant le SGT datent de la fin des années '60 (voir Shapiro et al., 1978) et le syndrome est encore considéré comme un trouble rare, notamment en Europe. Néanmoins, on assiste présentement à un foisonnement d'études étiologiques et cliniques dans la littérature scientifique. Le premier congrès mondial sur le SGT et les troubles tic a d'ailleurs eu lieu à Londres en 2015. Trois jours de conférences et de présentations multidisciplinaires faisant la synthèse des

Tableau 2

Étapes thérapeutiques des traitements CoPs (O'Connor, 2005) et Façotik (Leclerc et al., 2013)

\begin{tabular}{|c|c|c|}
\hline Étapes & Objectifs & Description \\
\hline 1 & $\begin{array}{l}\text { Entraînement à la prise de } \\
\text { conscience }\end{array}$ & $\begin{array}{l}\text { Évaluation des situations à haut/faible risque de } \\
\text { manifestation et du contexte relié aux tics }\end{array}$ \\
\hline 2 & $\begin{array}{l}\text { Entraînement à la } \\
\text { discrimination musculaire }\end{array}$ & $\begin{array}{l}\text { Contrôle de la tension musculaire par des } \\
\text { exercices de gradation }\end{array}$ \\
\hline 3 & $\begin{array}{l}\text { Entraînement à la } \\
\text { relaxation musculaire }\end{array}$ & Respiration et relaxation musculaire progressive \\
\hline 4 & $\begin{array}{l}\text { Réduction de l'activation } \\
\text { sensori-motrice }\end{array}$ & $\begin{array}{l}\text { Apprendre à se centrer sur les sensations } \\
\text { survenant avant ou pendant les tics }\end{array}$ \\
\hline 5 & $\begin{array}{l}\text { Analyse des styles de } \\
\text { planification de l'action }\end{array}$ & $\begin{array}{l}\text { Faire des liens entre les styles d'action, la tension } \\
\text { musculaire et les tics (p. ex., surpréparation, } \\
\text { suractivité) }\end{array}$ \\
\hline 6 & Restructuration cognitive & $\begin{array}{l}\text { Modification des attentes perfectionnistes } \\
\text { (frustration), des anticipations et des croyances } \\
\text { liées aux styles d'action }\end{array}$ \\
\hline 7 & $\begin{array}{l}\text { Restructuration } \\
\text { comportementale }\end{array}$ & $\begin{array}{l}\text { Apprentissage de comportements alternatifs qui } \\
\text { diminuent la tension musculaire globale }\end{array}$ \\
\hline 8 & $\begin{array}{l}\text { Restructuration cognitive et } \\
\text { comportementale globale }\end{array}$ & $\begin{array}{l}\text { Intégration des stratégies aux situations à haut } \\
\text { risque de manifestation }\end{array}$ \\
\hline 9 & Généralisation & $\begin{array}{l}\text { Appliquer les stratégies apprises à d'autres } \\
\text { situations }\end{array}$ \\
\hline 10 & Prévention de la rechute & \\
\hline
\end{tabular}


avancées scientifiques dans ce domaine précis. En plus de présenter les associations de soutien qui développent de plus en plus leur offre de services pour les personnes atteintes du SGT et qui participent au financement de certains projets de recherche (p. ex., Tourette Association of America, Tourette Action-UK, Tourette Canada), la programmation du congrès faisait état de deux milieux de recherche complémentaires; le domaine médical et les neurosciences, ainsi que le domaine de la santé mentale regroupant la psychologie et la psychiatrie. Les études fondamentales et cliniques issues de ces domaines de recherche reflètent des conceptions du SGT qui parfois s'opposent au sein de la communauté scientifique. Pourtant, que la conception que l'on ait du SGT soit médicale, psychiatrique ou comportementale, ces différents volets ne doivent pas être considérés indépendants ou isolés, mais bien complémentaires dans l'optique d'un modèle théorique élargi incluant l'ensemble des facteurs de la phénoménologie (génétique, neurobiologique, psychophysiologique, développemental, comportemental, cognitif et émotionnel). Un tel modèle conceptuel multimodal et intégré permettrait de considérer les composantes d'ordre psychologique et comportemental comme les médiateurs de la dysfonction neurodéveloppementale.

Malgré la recherche grandissante sur le sujet, une compréhension des causes exactes du SGT et des tics demeure encore incertaine, et ce, sans compter la présence quasi-systématique de troubles associés qui compliquent l'établissement des diagnostics différentiels et qui empêchent l'identification d'un profil neuropsychologique spécifique. Néanmoins, l'évaluation des fonctions cognitives des personnes ayant le SGT est recommandée, notamment lorsque des difficultés d'adaptation sociale, scolaire/académique et vocationnelle sont observées ou lorsque des déficits cognitifs ou des troubles d'apprentissage sont suspectés. De manière globale, la combinaison d'une évaluation médicale, neurospsychologique, comportementale (tics et symptômes associés) et émotionnelle (interférence au fonctionnement quotidien, qualité de vie) permettra de documenter les tics et de les distinguer d'autres comportements répétitifs. Elle permettra de mettre en place des mesures d'intervention et d'accommodation pour venir en aide le plus rapidement possible à l'individu aux prises avec un SGT. Parallèlement, il est essentiel que l'intervention soit d'approche multifactorielle, tel que recommandé dans les lignes directrices issues de méta-analyses (Murphy et al., 2013, Pringsheim et al., 2012; Verdellen et al., 2011;). Un traitement optimal devrait combiner la médication au besoin, ainsi qu'une thérapie cognitive et comportementale ciblant l'apprentissage de stratégies de gestion des tics. L'approche cognitive comportementale et psychophysiologique mise de l'avant par O'Connor (2005) (Leclerc et al., en préparation) représente une avenue en plein essor, puisqu'elle considère toute la complexité du SGT plutôt que de se centrer uniquement sur le tic. D'ailleurs, une étude 
par essais cliniques randomisés débutera sous peu au Centre d'études sur les troubles obsessionnels-compulsifs et les tics de Montréal afin de comparer l'effet de deux modalités de traitement visant à diminuer les tics (CBIT et CoPs) auprès d'un groupe d'enfants et d'un groupe d'adultes ${ }^{1}$. Les résultats de cette étude permettront d'une part de mieux comprendre et de mieux agir sur les manifestations en validant, le cas échéant, la nécessité d'intervenir sur les processus cognitifs et comportementaux sous-jacents aux tics et d'autre part, de vérifier l'effet du traitement non seulement sur la réduction des tics, mais aussi sur les déficits cognitifs que peuvent présenter l'enfant et l'adulte atteint du SGT. Des évaluations neuropsychologiques pré- et post-traitement offriront une meilleure compréhension du fonctionnement cognitif dans le SGT et permettront aussi d'évaluer l'impact du traitement ciblant les tics en ce qui a trait à la motricité fine, à la mémoire non verbale, à la fluidité verbale, ainsi qu'aux fonctions exécutives comme l'inhibition et la planification (p. ex., Como, 2001; Lavoie, Thibault \& O'Connor, 2012; Morand-Beaulieu, O'Connor, Sauvé, Blanchet, \& Lavoie, 2015; Lavoie, Thibault, Stip et O'Connor, 2007). Des résultats positifs sont attendus au plan clinique, cognitif et psychophysiologique. Ainsi, les données concernant la diminution des tics, les symptômes de troubles asociés, la qualité de vie et les profils cognitifs identifiés seront rapidement diffusés pour contribuer à réduire la souffrance marquée des personnes vivant avec un SGT et leur entourage. La diffusion des connaissances et une meilleure accessibilité aux services cliniques sont essentielles pour mieux soutenir et aider les personnes atteintes du SGT et leur famille.

\section{RÉFÉRENCES}

Abramovitch, A., Reese, H., Woods, D.W., Peterson, A., Deckersbach, T., Piacentini, J., ... \& Wilhelm, S. (2015). Psychometric properties of a self-report instrument for the assessment of tic severity in adults with tic disorders. Behavior Therapy, 46(6), 786-796,

Alsobrook, J. P. II, \& Pauls, D. L. (2002). A factor analysis of tic symptoms in Gilles de la Tourette's syndrome. The American Journal of Psychiatry, 159(2), 291-296.

American Psychiatric Association (2000). Diagnostic and statistical manual of mental disorders (Fourth edition, text revised). Washington, DC: American Psychiatric Publishing.

American Psychiatric Association. (2013). Diagnostic and statistical manual of mental disorders : DSM-5 ( $5^{\mathrm{e}}$ édition): Washington, DC : American Psychiatric Publishing.

Azrin, N. H., \& Nunn, R. G. (1973). Habit-reversal: A method of eliminating nervous habits and tics. Behaviour research and therapy, 11, 619-628.

Azrin, N. H., \& Peterson, A. L. (1988). Habit reversal for the treatment of Tourette syndrome. Behaviour research and therapy, 26, 347-351.

Budman, C. L., Rockmore, L., Stokes, J., \& Sossin, M. (2003). Clinical phenomenology of episodic rage in children with Tourette syndrome. Journal of Psychosomatic Research, 55(1), 59-65.

1. Subvention de fonctionnement des Instituts de recherche en santé du Canada“ Evaluation of a cognitive psychophysiological treatment for Tourette syndrome and tic disorders " (2015-2020). 
Capriotti, M. R., Espil, F. M., Conelea, C. A., \& Woods, D. W. (2013). Environmental factors as potential determinants of premonitory urge severity in youth with Tourette syndrome. Journal of Obsessive-Compulsive and Related Disorders, 2(1), 37-42.

Cavanna, A. E., Eddy, C., \& Rickards, H. E. (2009). Cognitive functioning in Tourette syndrome. Discovery medicine, 8(43), 191-195.

Chamberlain, S. R., Blackwell, A. D., Fineberg, N. A., Robbins, T. W., \& Sahakian, B. J. (2005). The neuropsychology of obsessive compulsive disorder: the importance of failures in cognitive and behavioural inhibition as candidate endophenotypic markers. Neuroscience \& Biobehavioral Reviews, 29(3), 399-419.

Chan, R. C., Shum, D., Toulopoulou, T., \& Chen, E. Y. (2008). Assessment of executive functions: Review of instruments and identification of critical issues. Archives of Clinical Neuropsychology, 23(2), 201-216.

Chang, S., Himle, M., Tucker, B., Woods, D., \& Piacentini, J. (2009). Initial development and psychometric properties of the Parent Tic Questionnaire (PTQ) to assess tic severity in children with chronic tic disorders. Child \& Family Behavior Therapy, 31(3), 181-191.

Channon, S., Gunning, A., Frankl, J., \& Robertson, M. M. (2006). Tourette's syndrome (TS): cognitive performance in adults with uncomplicated TS. Neuropsychology, 20(1), 58.

Clarke, M. A., Bray, M.A., Kehle, T. J., \& Truscott, S. D. (2001). A school-based intervention designed to reduce the frequency of tics in children with Tourette's syndrome. School psychology review, 30, 11-22.

Coffey, B. J. (2015). Complexities for assessment and treatment of co-occurring ADHD and tics. Current Developmental Disorders Reports, 2(4), 293-299.

Cohen, D. J., Leckman, J. F., \& Shaywitz, B. A. (1984). The Tourette's syndrome and other tics. In D. Shaffer, A. A. Ehrhardt, \& L. Greenhill (Éds), Diagnosis and treatment in pediatric psychiatry. New York, NY : Macmillan Free Press.

Como, P. G. (2001). Neuropsychological function in Tourette syndrome. Advances in Neurology, 85, 103-111.

Copur, M., Arpaci, B., Demir, T., \& Narin, H. (2007). Clinical effectiveness of quetiapine in children and adolescents with Tourette's Syndrome. Clinical Drug Investigation, 27(2), 123-130.

Crawford, S., Channon, S., \& Robertson, M. M. (2005). Tourette's syndrome: performance on tests of behavioural inhibition, working memory and gambling. Journal of Child Psychology and Psychiatry, 46(12), 1327-1336.

Debes, N. M., Lange, T., Jessen, T. L., Hjalgrim, H., \& Skov, L. (2011). Performance on Wechsler intelligence scales in children with Tourette syndrome. European Journal of Paediatric Neurology, 15(2), 146-154.

Deckersbach, T., Miller, K. K., Klibanski, A., Fischman, A., Dougherty, D. D., Blais, M. A., ... Rauch, S. L. (2006). Regional cerebral brain metabolism correlates of neuroticism and extraversion. Depression and Anxiety, 23, 133-138.

Dion, Y., Annable, L., Sandor, P., \& Chouinard, G. (2002). Risperidone in the treatment of Tourette's syndrome: a double-blind placebo-controlled trial. Journal of clinical psychopharmacology, 22, 31-39.

Drury, H., Channon, S., Barrett, R., Young, M. B., Stern, J. S., Simmons, H., \& Crawford, S. (2012). Emotional processing and executive functioning in children and adults with Tourette's syndrome. Child Neuropsychology, 18(3), 281-298.

Dufrene, B. A., Lestremau Harpole, L., Sterling, H. E., Perry, E. J., Burton, B., \& ZoderMartell, K. (2013). Functional analysis identified habit reversal components for the treatment of motor tics. Child et Family Behavior Therapy, 35(1), 41-62.

Eddy, C. M., \& Cavanna, A. E. (2013). Altered social cognition in Tourette syndrome: nature and implications. Behavioural Neurology, 27(1), 15-22.

Eddy, C. M., \& Cavanna, A. E. (2014). Set-shifting deficits a possible neurocognitive endophenotype for Tourette syndrome without ADHD. Journal of Attention Disorders (Epub ahead of print), doi: 10.1177/1087054714545536.

Eddy, C. M., Macerollo, A., Martino, D., \& Cavanna, A. E. (2015). Interpersonal reactivity differences in Tourette syndrome. Psychiatry Research, 228(3), 932-935.

Eddy, C. M., Rizzo, R., \& Cavanna, A. E. (2009). Neuropsychological aspects of Tourette syndrome: a review. Journal of Psychosomatic Research, 67(6), 503-513. 
Eddy, C. M., Rizzo, R., Gulisano, M., Agodi, A., Barchitta, M., Calì, P., \& Cavanna, A. E., (2011). Quality of life in young people with Tourette syndrome: a controlled study. Journal of Neurology, 258(2), 291-301.

Evers, R. F. A. \&, van de Wetering, B. J. M. (1994). A treatment model for motor tics based on a specific tension-reduction technique. Journal of Behavior Therapy and Experimental Psychiatry, 25, 255-260.

Fourneret, P., Desombre, H., \& Broussolle, E. (2014). Des tics moteurs au syndrome de Gilles de la Tourette: données actuelles, comorbidités et principes de prise en charge thérapeutique chez l'enfant et l'adolescent. Archives de pédiatrie, 21(6), 646-651.

Frank, M., \& Cavanna, A. E. (2012). Behavioural treatments for Tourette syndrome: An evidence-based review. Behavioural Neurology, 27, 105-117.

Freeman, R. D., Fast, D. K., Burd, L., Kerbeshian, J., Robertson, M. M., \& Sandor, P. (2000). An international perspective on Tourette syndrome: selected findings from 3500 individuals in 22 countries. Developmental Medicine \& Child Neurology, 42(7), 436-447.

Freeman, R. D., \& Tourette Syndrome International Database Consortium (2007). Tic disorders and ADHD: answers from a world-wide clinical dataset on Tourette syndrome. European Child \& Adolescent Psychiatry, 16(9), 15-23.

Gaffney, G., Sieg, K., \& Hellings, J. (1994). The MOVES: a self-rating scale for Tourette's syndrome. Journal of Children and Adolescence Psychopharmacologie, 4(4), 269-280.

Ganos, C., \& Martino, D. (2015). Tics and Tourette syndrome. Neurologic Clinics, 33(1), 115136.

Ganos, C., Roessner, V., \& Münchau, A. (2013). The functional anatomy of Gilles de la Tourette syndrome. Neuroscience \& Biobehavioral Reviews, 37(6), 1050-1062.

Ghanizadeh, A., \& Haghighi, A. (2014). Aripiprazole versus risperidone for treating children and adolescents with tic disorder: a randomized double blind clinical trial. Child Psychiatry and Human Development, 45(5), 596-603.

Gilbert, D. L., Batterson, R., Sethuraman, G., \& Sallee, F. R. (2004). Tic reduction with risperidone versus pimozide in a randomized, double-blind, crossover trial. Journal of American Academy of Child and Adolescent Psychiatry, 43, 206-214.

Hallett, M. (2015). Tourette Syndrome: Update. Brain and Development, 37(7), 651-655.

Hamel, N., O'Connor, K. P., Morand-Beaulieu, S., \& Lavoie, M. E. (2015). L'impulsivité et le syndrome de Gilles de la Tourette: état des connaissances. Revue québécoise de psychologie, 36(2), 53-79.

Harcherik, D. F., Leckman, J. F., Detlor, J., \& Cohen, D. J. (1984). A new instrument for clinical studies of Tourette's syndrome. Journal of the American Academy of Child Psychiatry, 23(2), 153-160.

Harris, E. L., Schuerholz, L. J., Singer, H. S., Reader, M. J., Brown, J. E., Cox, C., ... Denckla, M. B. (1995). Executive function in children with Tourette syndrome and/or attention deficit hyperactivity disorder. Journal of the International Neuropsychological Society, 1(06), 511-516.

Himle, M. B., Capriotti, M. R., Hayes, L. P., Ramanujam, K., Scahill, L., Sukhodolsky, D. G., ... Piacentini, J. (2014). Variables associated with tic exacerbation in children with chronic tic disorders. Behavior Modification, 38(2), 163-183.

Himle, M.B., \& Woods, D.W. (2005). An experimental evaluation of tic suppression and the tic rebound effect. Behaviour Research and Therapy, 43,1443-1451.

Hoekstra, P. J., Dietrich, A., Edwards, M. J., Elamin, I., \& Martino, D. (2013). Environmental factors in Tourette syndrome. Neuroscience \& Biobehavioral Reviews, 37(6), 1040-1049.

Hoogduin, K., Verdellen, C., \& Cath, D. (1997). Exposure and response prevention in the treatment of Gilles de la Tourette's syndrome: four case studies. Clinical Psychology and Psychotherapy, 4, 125-135.

Hwang, G. C., Tillberg, C. S., \& Scahill, L. (2012). Habit reversal training for children with Tourette syndrome: update and review. Journal of Child and Adolescent Psychiatric Nursing, 25, 178-183.

Jackson, G. M., Draper, A., Dyke, K., Pépés, S. E., \& Jackson, S. R. (2015). Inhibition, disinhibition, and the control of action in Tourette syndrome. Trends in Cognitive Sciences, 19(11), 655-665. 
Jankovic, J., \& Kurlan, R. (2011). Tourette syndrome: evolving concepts. Movement Disorders, 26(6), 1149-1156.

Kataoka, Y., Kalanithi, P. S., Grantz, H., Schwartz, M. L., Saper, C., Leckman, J. F., \& Vaccarino, F. M. (2010). Decreased number of parvalbumin and cholinergic interneurons in the striatum of individuals with Tourette syndrome. Journal of Comparative Neurology, 518(3), 277-291.

Lavoie, M. E., Leclerc, J., \& O'Connor, K. P. (2013). Bridging neuroscience and clinical psychology: cognitive behavioral and psychophysiological models in the evaluation and treatment of Gilles de la Tourette syndrome. Neuropsychiatry, 3(1), 75-87.

Lavoie, M. E., Leclerc, J., Thibault, G., \& O'Connor, K. P. (2012). Les interventions cognitivocomportementales auprès des enfants et des adolescents souffrant du syndrome Gilles de la Tourette et de tics chroniques. In S. Parent \& L. Turgeon (Éds), Intervention cognitivo-comportementale auprès des enfants et des adolescents: Troubles de comportement, vol. 2 (p. 87). Montréal : Presses de l'Université du Québec.

Lavoie, M. E., Thibault, G., Stip, E., \& O'Connor, K. P. (2007). Memory and executive functions in adults with Gilles de la Tourette syndrome and chronic tic disorder. Cognitive Neuropsychiatry, 12(2), 165-181.

Lebowitz, E. R., Motlagh, M. G., Katsovich, L., King, R. A., Lombroso, P. J., Grantz, H., ... Leckman, J. F. (2012). Tourette syndrome in youth with and without obsessive compulsive disorder and attention deficit hyperactivity disorder. European Child and Adolescent Psychiatry, 21(8), 451-457.

Leckman, J. F., Riddle, M. A., Hardin, M. T., Ort, S.I., Swartz, K. L., Stevenson, J., \& Cohen, D. J. (1989). The Yale Global Tic Severity Scale : Initial testing of a clinician-rated scale of tic severity. Journal of the American Academy of Child \& Adolescent Psychiatry, 28(4), 566-573.

Leclerc, J., Forget, J., \& O'Connor, K. P. (2008). Quand le corps fait à sa tête: le syndrome de Gilles de la Tourette. Éditions Multimondes.

Leclerc, J., Goulet, G., Hamel, N., \& O'Connor, K. P. (2013). Façotik; Léa et Nico font face aux tics. Guides méthodologiques (enfant et thérapeute). Document de travail, Centre d'études pour les troubles obsessionnels et les tics.

Leclerc, J. B., \& O'Connor, K. P. (2015, juin). Intervention in adults and children targeting underlined tics beliefs and processes. Communication orale présentée au 1er congrès mondial sur le trouble Tourette et les tics, Londres, Royaume-Uni.

Leclerc, J. B., Valois, P., J-Nolin, G., Bombardier, M., Ouellette, S., \& O'Connor, K.P. (en préparation). A therapy for tics in children managing underlying processes: A pilot study. Journal of Developmental and Physical Disabilities.

Lerner, A., Bagic, A., Simmons, J. M., Mari, Z., Bonne, O., Xu, B., ... \& Hallett, M. (2012). Widespread abnormality of the $\mathrm{y}$-aminobutyric acid-ergic system in Tourette syndrome. Brain, 135(Pt 6), 1926-1936.

Lyon, G. J., Samar, S., Jummani, R., Hirsch, S., Spirgel, A., Goldman, R., \& Coffey, B. J. (2009). Aripiprazole in children and adolescents with Tourette's disorder: an open-label safety and tolerability study. Journal of Child and Adolescent Psychopharmacology, 19(6), 623-633.

Matsuda, N., Kono, T., Nonaka, M., Shishikura, K., Konno, C., Kuwabara, H., ... Kano, Y. (2012). Impact of obsessive-compulsive symptoms in Tourette's syndrome on neuropsychological performance. Psychiatry and Clinical Neurosciences, 66(3), 195202.

Meidinger, A. L., Miltenberger, R. G., Himle, M., Omvig, M., Trainor, C., \& Crosby, R. (2005). An investigation of tic suppression and the rebound effect in Tourette's disorder. Behavior modification, 29, 716-745.

Meyers, J. E., \& Meyers, K. R. (1995). Rey complex figure test under four different administration procedures. The Clinical Neuropsychologist, 9(1), 63-67.

Morand-Beaulieu, S., O'Connor, K. P., Sauvé, G., Blanchet, P. J., \& Lavoie, M. E. (2015). Cognitive-behavioral therapy induces sensorimotor and specific electrocortical changes in chronic tic and Tourette's disorder. Neuropsychologia (Epub ahead of print), doi: 10.1016/j.neuropsychologia.2015.05.024 
Müller, S. V., Johannes, S., Wieringa, B., Weber, A., Müller-Vahl, K., Matzke, M., ... Münte, T. F. (2003). Disturbed monitoring and response inhibition in patients with Gilles de la Tourette syndrome and co-morbid obsessive compulsive disorder. Behavioural Neurology, 14(1-2), 29-37.

Murphy, T. K., Lewin, A. B., Storch, E. A., Stock, S., \& the American Academy of Child and Adolescent Psychiatry Committee on Quality Issues (2013). Practice parameter for the assessment and treatment of children and adolescents with tic disorders. Journal of the American Academy of Child and Adolescent Psychiatry, 52(12), 1341-1359.

Murphy, T., \& Muter, V. (2012). Risk factors for comorbidity in ADHD and GTS: looking beyond a single-deficit model. Applied Neuropsychology: Child, 1(2), 129-136.

Noton-Durand, F., \& Jalenques, I. (2008). Clinique du syndrome de Gilles de la Tourette et comorbidités psychiatriques associées chez l'enfant. Annales Médico-Psychologiques, revue psychiatrique, 166(4), 315-318.

O'Connor, K. P. (2002). A cognitive-behavioral / psychological model of tic disorders. Behaviour Research and Therapy, 40, 1113-1142.

O'Connor, K. P. (2005) Cognitive-behavioral management of tic disorders. Wiley, NY.

O'Connor, K. P., Brault, M., Loiselle, J., Robillard, S., Borgeat, F., \& Stip, E. (2001). Evaluation of a cognitive-behavioral program for the management of chronic tic and habit disorders. Behavior Research and Therapy, 39, 667-681.

O'Connor, K. P., Laverdure, A., Taillon, A., Stip, E., Borgeat, F., \& Lavoie, M. (2009). Cognitive behavioral management of Tourette's syndrome and chronic tic disorder in medicated and unmedicated samples. Behaviour Research and Therapy, 47, 10901095.

O'Connor, K. P., Lavoie, M. E., Stip, E., Borgeat, F., \& Laverdure, A. (2008). Cognitivebehaviour therapy and skilled motor performance in adults with chronic tic disorder. Neuropsychological Rehabilitation, 18(1), 45-64.

O'Connor, K. P., Lavoie, M. E., Blanchet, P., \& St-Pierre-Delorme, M.-E. (2015). Evaluation of a cognitive psychophysiological model for management of tic disorders: an open trial. The British Journal of Psychiatry (Epub ahead of print), doi: 10.1192/bjp.bp.114.154518

Paschou, P. (2013). The genetic basis of Gilles de la Tourette Syndrome. Neuroscience \& Biobehavioral Reviews, 37(6), 1026-1039.

Piacentini, J., Woods, D. W., Scahill, L., Wilhelm, S., Peterson, A. L., Chang, S., ... Walkup, J. T. (2010). Behavior therapy for children with Tourette disorder: a randomized controlled trial. Journal of the American Medical Association, 303(19), 1929-1937.

Pringsheim, T., Doja, A., Gorman, D., McKinlay, D., Day, L., Billinghurst, L., ... Sandor, P. (2012). Canadian guidelines for the evidence-based treatment of tic disorders: Pharmacotherapy. Canadian Journal of Psychiatry, 57(3), 133-143.

Robertson, M. M. (2000). Tourette syndrome, associated conditions and complexities of treatment. Brain, 123, 425-462.

Robertson, M. M., Althoff, R. R., Hafez, A., \& Pauls, D. L. (2008). Principal components analysis of a large cohort with Tourette syndrome. The British Journal of Psychiatry, 193(1), 31-36.

Roessner, V., Schoenefeld, K., Buse, J., Bender, S., Ehrlich, S., \& Münchau, A. (2013). Pharmacological treatment of tic disorders and Tourette Syndrome. Neuropharmacology, 68, 143-149.

Scahill, L., Erenberg, G., Berlin, C. M., Budman, C., Coffey, B. J., Jankovic, J., ... Tourette syndrome association medical advisory board: Practice committee. (2006). Contemporary assessment and pharmacotherapy of Tourette syndrome. NeuroRx, 3(2), 192-206.

Scharf, J. M., Miller, L. L., Mathews, C. A., \& Ben-Shlomo, Y. (2012). Prevalence of Tourette syndrome and chronic tics in the population-based Avon longitudinal study of parents and children cohort. Journal of the American Academy of Child \& Adolescent Psychiatry, 51(2), 192-201.

Serajee, F. J., \& Huq, A. M. (2015). Advances in Tourette syndrome: diagnosis and treatment. Pediatric Clinics of North America, 62(3), 571-820.

Shapiro A. K., Shapiro E., Bruun R. D., \& Swette R. D. (1978). Gilles de La Tourette syndrome. NY: Raven Press. 
Shytle, R. D., Silver, A. A., Sheehan, K. H., Wilkinson, B. J., Newman, M., Sanberg, P. R., \& Sheehan, D. (2003). The Tourette's Disorder Scale (TODS): development, reliability, and validity. Assessment, 10 (3), 273-287.

Steeves, T., McKinlay, B. D., Gorman, D., Billinghurst, L., Day, L., Carroll, A., ... Pringshelm, T. (2012). Canadian guidelines for the evidence-based treatment of tic disorders: Behavioural therapy, deep brain stimulation, and transcranial magnetic stimulation. Canadian Journal of Psychiatry, 57(3), 144-151.

Storch, E. A., Lack, C. W., Simons, L. E., Goodman, W. K., Murphy, T. K., \& Geffken, G. R. (2007). A Measure of functional impairment in youth with Tourette's syndrome. Journal of Pediatric Psychology, 32(8), 950-959.

Sukhodolsky, D. G., Scahill, L., Zhang, H., Peterson, B. S., King, R.A., Lombroso, P. J., ... Leckman, J.F. (2003). Disruptive behavior in children with Tourette syndrome: Association with ADHD comorbidity, tic severity, and functional impairment. Journal of the American Academy of Child and Adolescent Psychiatry, 42(1), 98-105.

Thibault, G., Felezeu, M., O'Connor, K. P., Todorov, C., Stip, E., \& Lavoie, M. E. (2008). Influence of comorbid obsessive-compulsive symptoms on brain event-related potentials in Gilles de la Tourette syndrome. Progress in Neuro-Psychopharmacology and Biological Psychiatry, 32(3), 803-815.

Verdellen, C. W. J., Keijsers, G. P. J., Cath, D. C., \& Hoogduin, C. A. L. (2004). Exposure with response prevention versus habit reversal in Tourette's syndrome. Behaviour Research and Therapy, 42, 501-511.

Verdellen, C., van de Griendt, J., Hartmann, A., Murphy, T., \& ESSTS Guidelines Group. (2011). European clinical guidelines for Tourette syndrome and other tic disorders. Part III: behavioural and psychosocial interventions. European Child and Adolescent Psychiatry, 20(4), 197-207.

Vicario, C. M., Gulisano, M., Martino, D., \& Rizzo, R. (2015). Timing recalibration in childhood Tourette syndrome associated with persistent pimozide treatment. Journal of Neuropsychology.

Wang, Z., Maia, T. V., Marsh, R., Colibazzi, T., Gerber, A., \& Peterson, B. S. (2014). The neural circuits that generate tics in Tourette's syndrome. American Journal of Psychiatry, 168(12), 1326-1337.

Watson, T. S., Howell, L. A., \& Smith, S. L. (2001). Behavioral interventions for tic disorders. In Woods, D. W., \& Miltenberger, R. G. (Eds), Tic disorders, trichotillomania, and other repetitive behavior disorders, behavioral approaches to analysis and treatment (pp. 7396). Boston: Kluwer academic publishers.

Wilhelm, S., Deckersbach, T., Coffey, B. J., Bohne, A., Peterson, A. L., \& Baer, L. (2003). Habit reversal versus supportive psychotherapy for Tourette's disorder: a randomized controlled trial. The American Journal of Psychiatry, 160, 1175-1177.

Woods, D. W., \& Miltenberger, R. G. (1995). Habit reversal: a review of applications. Journal of Behaviour Therapy and Experimental Psychiatry, 26, 123-131.

Woods, D. W., Piacentini, J., Chang, S., Deckersbach, T., Ginsburg, G., \& Peterson, A. (2008). Managing Tourette syndrome: a behavioral intervention for children and adults therapist guide: a behavioral intervention for children and adults therapist guide: Oxford University Press.

Woods, D. W., Piacentini, J. C., Scahill, L., Peterson, A. L., Wilhelm, S., Chang, S., ... Walkup, J. T., (2011). Behavior therapy for tics in children: acute and long-term effects on psychiatric and psychosocial functioning. Journal of Child Neurology, 26(7), 858-865.

Worbe, Y., Lehericy, S., \& Hartmann, A. (2015). Neuroimaging of tic genesis: Present status and future perspectives. Movement Disorders, 30(9), 1179-1183.

Yoo, H. K., Lee, J.-S., Paik, K.-W., Choi, S.-H., Yoon, S. J., Kim, J. E., \& Hong, J. P. (2011). Open-label study comparing the efficacy and tolerability of aripiprazole and haloperidol in the treatment of pediatric tic disorders. European Child et Adolescent Psychiatry, 20(3), 127-135. 
Syndrome Gilles de la Tourette

\section{RÉSUMÉ}

L'objet de cet article est de présenter les avancées scientifiques concernant le syndrome de Gilles de la Tourette (SGT), dans l'optique où il fait partie de la nouvelle section des troubles neurodéveloppementaux du DSM-5. La définition et la symptomatologie des troubles tic, et particulièrement du SGT, sont présentées. Les troubles associés, ainsi que l'évaluation diagnostique et différentielle sont abordés sous l'angle clinique et neuropsychologique. Enfin, les traitements recommandés, et s'appuyant sur des données probantes, sont présentés de manière à outiller les professionnels et les psychologues, tout en les informant des principaux modèles conceptuels permettant une meilleure compréhension clinique des tics.

\section{MOTS CLÉS}

Tourette, tics, évaluation, traitement, compréhension clinique

\section{ABSTRACT}

The object of this article is to present scientific advances on Tourette disorder (TD) in regards to the new section of the DSM-5" neurodevelopmental disorders". The definition and symptomatology of tics disorders, and particularly TD, are presented. The comorbidities, as well as the diagnostic and differential evaluation are presented under a clinical and a neuropsychological point of view. Finally, recommended treatments and evidence-based practices are presented to guide professionals and psychologists, as well as to inform them about the principals conceptual models leading to a better clinical understanding of tics.

\section{KEY WORDS}

Tourette, tics, evaluation, treatment, clinical comprehension 